\title{
Composition iterates, Cauchy, translation, and Sincov inclusions
}

\section{Włodzimierz Fechner}

Institute of Mathematics,

Lodz University of Technology, 90-924 Łódź, Poland

email: wlodzimierz.fechner@p.lodz.pl

\author{
Árpád Száz \\ Institute of Mathematics, \\ University of Debrecen, \\ H-4002 Debrecen, Hungary \\ email: szaz@science.unideb.hu
}

Abstract. Improving and extending some ideas of Gottlob Frege from 1874 (on a generalization of the notion of the composition iterates of a function), we consider the composition iterates $\varphi^{n}$ of a relation $\varphi$ on $X$, defined by

$$
\varphi^{0}=\Delta_{X}, \quad \varphi^{n}=\varphi \circ \varphi^{n-1} \quad \text { if } \quad n \in \mathbb{N}, \quad \text { and } \quad \varphi^{\infty}=\bigcup_{n=0}^{\infty} \varphi^{n} .
$$

In particular, by using the relational inclusion $\varphi^{\mathrm{n}} \circ \varphi^{\mathrm{m}} \subseteq \varphi^{\mathrm{n}+\mathrm{m}}$ with $\mathrm{n}, \mathrm{m} \in \overline{\mathbb{N}}_{0}=\{0\} \cup \mathbb{N} \cup\{\infty\}$, we show that the function $\alpha$, defined by

$$
\alpha(n)=\varphi^{n} \quad \text { for } n \in \overline{\mathbb{N}}_{0},
$$

satisfies the Cauchy problem

$$
\alpha(n) \circ \alpha(m) \subseteq \alpha(n+m), \quad \alpha(0)=\Delta_{X} .
$$

Moreover, the function $\mathrm{f}$, defined by

$$
f(n, A)=\alpha(n)[A] \quad \text { for } \quad n \in \overline{\mathbb{N}}_{0} \quad \text { and } \quad A \subseteq X,
$$

2010 Mathematics Subject Classification: Primary 39B12, 39B52, 39B62; Secondary 20N02, 26E25, 54E25

Key words and phrases: relations, groupoids, composition iterates, Cauchy, translation and Sincov inclusions 
satisfies the translation problem

$$
f(n, f(m, A)) \subseteq f(n+m, A), \quad f(0, A)=A .
$$

Furthermore, the function $\mathrm{F}$, defined by

$$
F(A, B)=\left\{n \in \overline{\mathbb{N}}_{0}: \quad A \subseteq f(n, B)\right\} \quad \text { for } \quad A, B \subseteq X,
$$

satisfies the Sincov problem

$$
\mathrm{F}(\mathrm{A}, \mathrm{B})+\mathrm{F}(\mathrm{B}, \mathrm{C}) \subseteq \mathrm{F}(\mathrm{A}, \mathrm{C}), \quad 0 \in \mathrm{F}(\mathrm{A}, \mathrm{A}) .
$$

Motivated by the above observations, we investigate a function $\mathrm{F}$ on the product set $\mathrm{X}^{2}$ to the power groupoid $\mathcal{P}(\mathrm{U})$ of an additively written groupoid $\mathrm{U}$ which is supertriangular in the sense that

$$
F(x, y)+F(y, z) \subseteq F(x, z)
$$

for all $x, y, z \in X$. For this, we introduce the convenient notations

$$
R(x, y)=F(y, x) \quad \text { and } \quad S(x, y)=F(x, y)+R(x, y)
$$

and

$$
\Phi(x)=F(x, x) \quad \text { and } \quad \Psi(x)=\bigcup_{y \in X} S(x, y)
$$

Moreover, we gradually assume that $\mathrm{U}$ and $\mathrm{F}$ have some useful additional properties. For instance, $\mathrm{U}$ has a zero, $\mathrm{U}$ is a group, $\mathrm{U}$ is commutative, $\mathrm{U}$ is cancellative, or $\mathrm{U}$ has a suitable distance function; while $\mathrm{F}$ is nonpartial, $\mathrm{F}$ is symmetric, skew symmetric, or single-valued.

\section{A few basic facts on relations}

In [40], a subset $F$ of a product set $X \times Y$ is called a relation on $X$ to $\mathrm{Y}$. In particular, a relation on $X$ to itself is called a relation on $X$. More specially, $\Delta_{X}=\{(x, x): x \in X\}$ is called the identity relation on $X$.

If $F$ is a relation on $X$ to $Y$, then by the above definitions we can also state that $F$ is a relation on $X \cup Y$. However, for our present purposes, the latter view of the relation $F$ would also be quite unnatural.

If $F$ is a relation on $X$ to $Y$, then for any $x \in X$ and $A \subseteq X$ the sets $F(x)=\{y \in Y:(x, y) \in F\}$ and $F[A]=\bigcup_{a \in A} F(a)$ are called the images of $x$ and $A$ under $F$, respectively.

If $(x, y) \in F$, then instead of $y \in F(x)$, we may also write $x F y$. However, instead of $F[A]$, we cannot write $F(A)$. Namely, it may occur that, in addition to $A \subseteq X$, we also have $A \in X$. 
The sets $D_{F}=\{x \in X: F(x) \neq \emptyset\}$ and $R_{F}=F[X]$ are called the domain and range of $\mathrm{F}$, respectively. If in particular $\mathrm{D}_{\mathrm{F}}=\mathrm{X}$, then we say that $\mathrm{F}$ is a relation of $\mathrm{X}$ to $\mathrm{Y}$, or that $\mathrm{F}$ is a nonpartial relation on $\mathrm{X}$ to $\mathrm{Y}$.

In particular, a relation $f$ on $X$ to $Y$ is called a function if for each $x \in D_{f}$ there exists $y \in Y$ such that $f(x)=\{y\}$. In this case, by identifying singletons with their elements, we may simply write $f(x)=y$ instead of $f(x)=\{y\}$.

In particular, a function $\star$ of $\mathrm{X}$ to itself is called a unary operation on $\mathrm{X}$, while a function $*$ of $X^{2}$ to $X$ is called a binary operation on $X$. In this case, for any $x, y \in X$, we usually write $x^{\star}$ and $x * y$ instead of $\star(x)$ and $*((x, y))$.

If $F$ is a relation on $X$ to $Y$, then we can easily see that $F=\bigcup_{x \in X}\{x\} \times F(x)$. Therefore, the values $F(x)$, where $x \in X$, uniquely determine $F$. Thus, a relation $F$ on $X$ to $Y$ can also be naturally defined by specifying $F(x)$ for all $x \in X$.

For instance, the inverse $F^{-1}$ can be defined such that $F^{-1}(y)=\{x \in X: y \in$ $F(x)\}$ for all $y \in Y$. Moreover, if $G$ is a relation on $Y$ to $Z$, then the composition $\mathrm{G} \circ \mathrm{F}$ can be defined such that $(\mathrm{G} \circ \mathrm{F})(\mathrm{x})=\mathrm{G}[\mathrm{F}(\mathrm{x})]$ for all $x \in X$.

If $F$ is a relation on $X$ to $Y$, then a relation $\Phi$ of $D_{F}$ to $Y$ is called a selection relation of $\mathrm{F}$ if $\Phi \subseteq \mathrm{F}$, i.e., $\Phi(x) \subseteq \mathrm{F}(\mathrm{x})$ for all $x \in \mathrm{D}_{\mathrm{F}}$. By using the Axiom of Choice, it can be seen that every relation is the union of its selection functions.

For a relation $F$ on $X$ to $Y$, we may naturally define two set-valued functions $\varphi$ of $\mathrm{X}$ to $\mathcal{P}(\mathrm{Y})$ and $\Phi$ of $\mathcal{P}(\mathrm{X})$ to $\mathcal{P}(\mathrm{Y})$ such that $\varphi(\mathrm{x})=\mathrm{F}(\mathrm{x})$ for all $\mathrm{x} \in \mathrm{X}$ and $\Phi(A)=F[A]$ for all $A \subseteq X$.

Functions of $X$ to $\mathcal{P}(Y)$ can be identified with relations on $X$ to $Y$, while functions of $\mathcal{P}(\mathrm{X})$ to $\mathcal{P}(\mathrm{Y})$ are more powerful objects than relations on $\mathrm{X}$ to $\mathrm{Y}$. They were briefly called co-relations on $\mathrm{X}$ to $\mathrm{Y}$ in [40].

In particular, a relation $\mathrm{R}$ on $\mathrm{X}$ can be briefly defined to be reflexive if $\Delta_{\mathrm{X}} \subseteq \mathrm{R}$, and transitive if $\mathrm{R} \circ \mathrm{R} \subseteq \mathrm{R}$. Moreover, $\mathrm{R}$ can be briefly defined to be symmetric if $\mathrm{R}^{-1} \subseteq \mathrm{R}$, and antisymmetric if $\mathrm{R} \cap \mathrm{R}^{-1} \subseteq \Delta_{X}$.

Thus, a reflexive and transitive (symmetric) relation may be called a preorder (tolerance) relation, and a symmetric (antisymmetric) preorder relation may be called an equivalence (partial order) relation.

For $A \subseteq X$, Pervin's relation $R_{A}=A^{2} \cup A^{c} \times X$, with $A^{c}=X \backslash A$, is an important preorder on $X$. While, for a pseudometric $\mathrm{d}$ on $\mathrm{X}$, Weil's surrounding $B_{r}=\left\{(x, y) \in X^{2}: d(x, y)<r\right\}$, with $r>0$, is an important tolerance on $X$.

Note that $S_{A}=R_{A} \cap R_{A}^{-1}=R_{A} \cap R_{A^{c}}=A^{2} \cap\left(A^{c}\right)^{2}$ is already an equivalence on $X$. And, more generally if $\mathcal{A}$ is a partition of $X$, then $S_{\mathcal{A}}=\bigcup_{A \in \mathcal{A}} A^{2}$ is an equivalence on $\mathrm{X}$ which can, to some extent, be identified with $\mathcal{A}$. 


\section{A few basic facts on ordered sets and groupoids}

If $\leq$ is a relation on $X$, then motivated by Birkhoff $[5$, p. 1] the ordered pair $X(\leq)=(X, \leq)$ is called a goset (generalized ordered set) [39]. In particular, it is called a proset (preordered set) if the relation $\leq$ is a preorder on $\mathrm{X}$.

Quite similarly, a goset $X(\leq)$ is called a poset (partially ordered set) if the relation $\leq$ is a partial order on $X$. The importance of posets lies mainly in the fact that any family of sets forms a poset with set inclusion.

A function $f$ of one goset $X(\leq)$ to another $Y(\leq)$ is called increasing if $x_{1} \leq x_{2}$ implies $f\left(x_{1}\right) \leq f\left(x_{2}\right)$ for all $x_{1}, x_{2} \in X$. The function $f$ can now be briefly called decreasing if it is increasing as a function of $\mathrm{X}(\leq)$ to the dual $\mathrm{Y}(\geq)$.

An increasing function $\varphi$ of the goset $X=X(\leq)$ to itself is called a projection (involution) operation on $\mathrm{X}$ if it is idempotent (involutive) in the sense that $\varphi \circ \varphi=\varphi\left(\varphi \circ \varphi=\Delta_{X}\right)$. Note that $\varphi \circ \varphi=\Delta_{X}$ if and only if $\varphi^{-1}=\varphi$.

Moreover, a projection operation $\varphi$ on a poset $X$ is called a closure operation on $\mathrm{X}$ if it is extensive in the sense that $\Delta_{\mathrm{X}} \leq \varphi$. That is, $\chi \leq \varphi(\mathrm{x})$ for all $\chi \in \mathrm{X}$. The interior operations can again be most briefly defined by dualization.

If $f$ is a function of one goset $X$ to another $Y$ and $g$ is a function of $Y$ to $X$ such that, for any $x \in X$ and $y \in Y$, we have $f(x) \leq y$ if and only $x \leq g(y)$, then $\mathrm{g}$ is called a Galois adjoint of $\mathrm{f}[12, \mathrm{p} .155]$.

Hence, by taking $\varphi=g \circ f$, one can easily see that, for any $u, v \in X$, we have $f(u) \leq f(v)$ if and only if $u \leq \varphi(v)$. Moreover, if $X$ and $Y$ are prosets, then it can be shown that $f$ is increasing, $\varphi$ is a closure and $f=f \circ \varphi$ [39].

If + is a binary operation on a set $X$, then the ordered pair $X(+)=(X,+)$ is called an additive groupoid. Recently, groupoids are usually called magmas, not to be confused with Brandt groupoids [6].

If $X$ is a groupoid, then for any $A, B \subseteq X$ we may also naturally define $A+B=\{x+y: x \in A, y \in B\}$. Thus, by identifying singletons with their elements, $X$ may be considered as a subgoupoid of its power groupoid $\mathcal{P}(X)$.

In a groupoid $X$, for any $n \in \mathbb{N}$ and $x \in X$ we may also naturally define $n x=x$ if $n=1$, and $n x=(n-1) x+x$ if $n>1$. Thus, for any $n \in \mathbb{N}$ and $A \subseteq X$, we may also naturally define $n A=\{n x: x \in A\}$.

If $X$ is a semigroup (associative groupoid), then we have $(n+m) x=n x+m x$ and $(n m) x=n(m x)$ for all $n, m \in \mathbb{N}$ and $x \in X$. However, the equality $n(x+y)=n x+n y$ requires the elements $x, y \in X$ to be commuting [19].

If the groupoid $X$ has a zero element 0 , then we also naturally define $0 x=0$ for all $x \in X$. Moreover, if $X$ is a group, then we also naturally define $(-\mathfrak{n}) x=$ $n(-x)$ for all $n \in \mathbb{N}$ and $x \in X$. And thus also $k A$ for all $k \in \mathbb{Z}$ and $A \subseteq X$.

Concerning the corresponding operations in $\mathcal{P}(X)$, we must be very careful. 
Namely, in general, we only have $(n+m) A \subseteq n A+m A$ and $n A \subseteq \sum_{i=1}^{n} A$ for all $n, m \in \mathbb{N}$ and $A \subseteq X$. However, $\mathcal{P}(X)$ has a richer structure than $X$.

In particular, an element $x$ of a groupoid $X$ is called left-cancellable if $x+y=$ $x+z$ implies $y=z$ for all $y, z \in X$. Moreover, the groupoid $X$ is called leftcancellative if every element of $\mathrm{X}$ is left-cancellable.

"Right-cancellable" and "right-cancellative" are to be defined quite similarly. Moreover, for instance, the groupoid $\mathrm{X}$ is to be called cancellative if it is both left-cancellative and right-cancellative.

A semigroup $X$ can be easily embedded in a monoid (semigroup with zero element), by adjoining an element 0 not in $X$, and defining $0+x=x+0=x$ for all $x \in X$. Important monoids will be $\mathbb{N}_{0}=\{0\} \cup \mathbb{N}$ and $\overline{\mathbb{N}}_{0}=\mathbb{N}_{0} \cup\{\infty\}$.

\section{The finite composition iterates of a relation}

Notation 1 In the sequel, we shall assume that $\mathrm{X}$ is a set, $\Delta$ is the identity function of $\mathrm{X}$ and $\varphi$ is a relation on $\mathrm{X}$.

Note that the family $\mathcal{P}\left(\mathrm{X}^{2}\right)$ of all relations on $\mathrm{X}$ forms a semigroup, with identity element $\Delta$, with respect to the composition of relations. Therefore, we may naturally use the following

Definition 1 Define $\varphi^{0}=\Delta$, and for any $n \in \mathbb{N}$

$$
\varphi^{\mathfrak{n}}=\varphi \circ \varphi^{\mathrm{n}-1} .
$$

Remark 1 Thus, for each $n \in \mathbb{N}_{0}, \varphi^{n}$ is also a relation on $X$ which is called the nth composition iterate of $\varphi$.

Now, as a particular case of a more general theorem on monoids, we can state the following theorem whose direct proof is included here only for the reader's convenience.

Theorem 1 For any $\mathrm{n}, \mathrm{m} \in \mathbb{N}_{0}$, we have

$$
\varphi^{\mathrm{n}+\mathrm{m}}=\varphi^{\mathrm{n}} \circ \varphi^{\mathrm{m}} .
$$

Proof. For fixed $m \in \mathbb{N}_{0}$, we shall prove, by induction, that

$$
\varphi^{\mathrm{m}+\mathrm{n}}=\varphi^{\mathrm{n}} \circ \varphi^{\mathrm{m}}
$$


for all $n \in \mathbb{N}_{0}$. Hence, by the commutativity of the addition in $\mathbb{N}_{0}$, the assertion of the theorem follows.

By Definition 1, we evidently have $\varphi^{\mathrm{m}+0}=\varphi^{\mathrm{m}}=\Delta \circ \varphi^{\mathrm{m}}=\varphi^{0} \circ \varphi^{\mathrm{m}}$. Therefore, the required equity is true for $n=0$.

Let us suppose now that the required equality is true for some $n \in \mathbb{N}_{0}$. Then, by Definition 1, the above assumption, and the corresponding associativities, we have

$$
\begin{aligned}
\varphi^{\mathrm{m}+(\mathfrak{n}+1)} & =\varphi^{(\mathrm{m}+\mathfrak{n})+1}=\varphi \circ \varphi^{\mathrm{m}+\mathrm{n}} \\
& =\varphi \circ\left(\varphi^{\mathfrak{n}} \circ \varphi^{\mathrm{m}}\right)=\left(\varphi \circ \varphi^{\mathfrak{n}}\right) \circ \varphi^{\mathrm{m}}=\varphi^{\mathfrak{n}+1} \circ \varphi^{\mathrm{m}} .
\end{aligned}
$$

Therefore, the required equality is also true for $n+1$.

Remark 2 This theorem shows that the family $\left\{\varphi^{n}\right\}_{\mathfrak{n}=0}^{\infty}$ also forms a semigroup, with identity element $\Delta$, with respect to composition.

By induction, we can also easily prove the less trivial part of the following

Theorem 2 The following assertions are equivalent:

(1) $\Delta \subseteq \varphi$;

(2) $\varphi^{\mathfrak{n}} \subseteq \varphi^{\mathrm{n}+1}$ for all $\mathrm{n} \in \mathbb{N}_{0}$.

Remark 3 This theorem shows that the sequence $\left(\varphi^{n}\right)_{n=0}^{\infty}$ is increasing, with respect to set inclusion, if and only if the relation $\varphi$ is reflexive on $X$.

Note that if in particular $\varphi$ is reflexive on $X$ and $\varphi$ is a function, then we necessarily have $\varphi=\Delta$, and thus also $\varphi^{n}=\Delta$ for all $n \in \mathbb{N}_{0}$.

Therefore, in the important particular case when $\varphi$ is a function of $X$ to itself, Theorem 2 cannot have any significance.

\section{The infinite composition iterate of a relation}

In addition to Definition 1, we may also naturally use the following

Definition 2 Define

$$
\varphi^{\infty}=\bigcup_{n=0}^{\infty} \varphi^{n}
$$

Remark 4 Moreover, the relations

$$
\varliminf_{n \rightarrow \infty} \varphi^{n}=\bigcup_{n=0}^{\infty} \bigcap_{k=n}^{\infty} \varphi^{k} \quad \text { and } \quad \varlimsup_{n \rightarrow \infty} \varphi^{n}=\bigcap_{n=0}^{\infty} \bigcup_{k=n}^{\infty} \varphi^{k}
$$


may also be naturally investigated.

Note that if in particular the sequence $\left(\varphi^{n}\right)_{n=0}^{\infty}$ is increasing with respect to set inclusion, then these relations coincide with $\varphi^{\infty}$.

The relation $\varphi^{\infty}$ is called the preorder hull (closure) of $\varphi$. Namely, we have

Theorem $3 \varphi^{\infty}$ is the smallest preorder relation on $\mathrm{X}$ containing $\varphi$.

Proof. By Definition 2, it is clear that $\Delta \subseteq \varphi^{\infty}$ and $\varphi \subseteq \varphi^{\infty}$. Thus, $\varphi^{\infty}$ is reflexive and contains $\varphi$.

Moreover, if $(x, y) \in \varphi^{\infty}$ and $(y, z) \in \varphi^{\infty}$, then by Definition 2 there exist $\mathrm{m}, \mathrm{n} \in \mathbb{N}_{0}$ such that $(x, y) \in \varphi^{\mathrm{m}}$ and $(y, z) \in \varphi^{\mathfrak{n}}$. Hence, by using Theorem 1 , we can infer that $(x, z) \in \varphi^{\mathfrak{n}} \circ \varphi^{\mathrm{m}}=\varphi^{\mathrm{n}+\mathrm{m}}$. Thus, by Definition 2 , we also have $(x, z) \in \varphi^{\infty}$. Therefore, $\varphi^{\infty}$ is also transitive.

On the other hand, if $\psi$ is a relation on $X$ such that $\varphi \subseteq \psi$, then we can note that $\varphi^{n} \subseteq \psi^{n}$ for all $n \in \mathbb{N}_{0}$, and thus by Definition 2 we have $\varphi^{\infty} \subseteq \psi^{\infty}$. Moreover, if $\psi$ is reflexive, then $\psi^{0} \subseteq \psi$. And, if $\psi$ is transitive, then $\psi^{n} \subseteq \psi$ for all $n \in \mathbb{N}$. Therefore, if $\psi$ is both reflexive and transitive, then by Definition 2 we have $\psi^{\infty} \subseteq \psi$, and thus also $\varphi^{\infty} \subseteq \psi$.

Now, as an immediate consequence of this theorem, we can also state

Corollary 1 The following assertions are equivalent:

(1) $\varphi^{\infty}=\varphi$;

(2) $\varphi$ is a preorder on $\mathrm{X}$.

Remark 5 From the above results, it is clear that $\infty$ is a closure operation on the poset $\mathcal{P}\left(\mathrm{X}^{2}\right)$.

In general, it is not even finitely union preserving. However, it is compatible with the inversion of relations [18].

Moreover, in addition to Theorem 1, we can also easily prove the following

Theorem 4 For any $\mathrm{n}, \mathrm{m} \in \overline{\mathbb{N}}_{0}$, we have

$$
\varphi^{\mathrm{n}} \circ \varphi^{\mathrm{m}} \subseteq \varphi^{\mathrm{n}+\mathrm{m}} .
$$

Moreover, if $\varphi$ is reflexive on $\mathrm{X}$, then the corresponding equality is also true.

Proof. If in particular $n, m \in \mathbb{N}_{0}$, then by Theorem 1 the corresponding equality is true even if $\varphi$ is not assumed to be reflexive.

Moreover, by using Definition 2 and Theorem 3, we can see that

$$
\varphi^{\mathrm{n}} \circ \varphi^{\infty} \subseteq \varphi^{\infty} \circ \varphi^{\infty} \subseteq \varphi^{\infty}=\varphi^{\mathrm{n}+\infty} .
$$


Furthermore, if $\varphi$ is reflexive, then it is clear that we also have

$$
\varphi^{\infty}=\Delta \circ \varphi^{\infty} \subseteq \varphi^{\mathrm{n}} \circ \varphi^{\infty} .
$$

Therefore, in this case, $\varphi^{n} \circ \varphi^{\infty}=\varphi^{\infty}=\varphi^{\mathrm{n}+\infty}$ also holds. The case " $\infty+\mathrm{m}^{\prime}$ can be treated quite similarly.

Remark 6 Now, in addition to Theorem 2 , we can only state that $\varphi^{\mathfrak{n}} \subseteq \varphi^{\infty}$ for all $n \in \overline{\mathbb{N}}_{0}$.

However, by [30], we may naturally say that $\varphi$ is $n$-well-chained if $\varphi^{n}=X^{2}$. And, $\varphi$ is $\mathrm{n}$-connected if $\varphi \cup \varphi^{-1}$ is $\mathrm{n}$-well-chained.

Moreover, under the notation $\mathcal{T}_{\varphi}=\{A \subseteq X: \varphi[A] \subseteq A\}$ of [24], we have $\varphi^{\infty}=\bigcap_{A \in \mathcal{T}_{\varphi}} R_{A}$. And, $\varphi^{\infty}$ is the largest relation on $X$ such that $\mathcal{T}_{\varphi}=\mathcal{T}_{\varphi}$.

\section{From the composition iterates to a Cauchy inclu- sion}

Now, extending an idea of Frege $[15,16]$, we may also naturally introduce

Definition 3 For any $n \in \overline{\mathbb{N}}_{0}$, define

$$
\alpha(n)=\varphi^{n} .
$$

Thus, $\alpha$ may be considered as a relation on $\overline{\mathbb{N}}_{0}$ to $X^{2}$, or as a function of $\overline{\mathbb{N}}_{0}$ to $\mathcal{P}\left(\mathrm{X}^{2}\right)$, which can be proved to satisfy a Cauchy type inclusion.

First of all, by Theorem 1, we evidently have the following

Theorem 5 For any $\mathrm{n}, \mathrm{m} \in \mathbb{N}_{0}$, we have

$$
\alpha(n+m)=\alpha(n) \circ \alpha(m) .
$$

Proof. By Definition 3 and Theorem 1, it is clear that

$$
\alpha(n+m)=\varphi^{n+m}=\varphi^{n} \circ \varphi^{m}=\alpha(n) \circ \alpha(m) .
$$

Remark 7 In addition to this theorem, it is also worth noticing that $\alpha(0)=\Delta$.

Moreover, by Theorem 2, we can also at once state the following 
Theorem 6 The following assertions are equivalent:
(1) $\Delta \subseteq \varphi$;
(2) $\alpha(\mathrm{n}) \subseteq \alpha(\mathrm{n}+1)$ for all $\mathrm{n} \in \mathbb{N}_{0}$.

Remark 8 Thus, the restriction of the set-valued function $\alpha$ to $\mathbb{N}_{0}$ is increasing, with respect to set inclusion, if and only if the relation $\varphi$ is reflexive on $X$.

By using Theorem 4 instead of Theorem 1, we can also easily establish

Theorem 7 For any $\mathrm{n}, \mathrm{m} \in \overline{\mathbb{N}}_{0}$ we have

$$
\alpha(n) \circ \alpha(m) \subseteq \alpha(n+m) .
$$

Moreover, if $\varphi$ is reflexive on $\mathrm{X}$, then the corresponding equality is also true.

Remark 9 Now, in addition to Theorem 6 , we can also state that $\alpha(\mathrm{n}) \subseteq$ $\alpha(\infty)$ for all $n \in \overline{\mathbb{N}}_{0}$.

Thus, in particular, the set-valued function $\alpha$ is increasing, with respect to set inclusion, if and only if the relation $\varphi$ is reflexive on $X$.

\section{From a Cauchy inclusion to a translation inclu- sion}

Now, as an extension of our former observations, we may naturally start with

Notation 2 Suppose that $\mathrm{U}$ is a additive groupoid and $\alpha$ is a relation on $\mathrm{U}$ to $\mathrm{X}^{2}$ such that

$$
\alpha(u) \circ \alpha(v) \subseteq \alpha(u+v)
$$

for all $u, v \in \mathrm{U}$.

Thus, extending an idea of Frege $[15,16]$, we may also naturally introduce

Definition 4 For any $u \in U$ and $A \subseteq X$, define

$$
f(u, A)=\alpha(u)[A] .
$$

Thus, $f$ may be considered a relation on $U \times \mathcal{P}(X)$ to $X$, or as a function of $\mathrm{U} \times \mathcal{P}(\mathrm{X})$ to $\mathcal{P}(\mathrm{X})$, which can be proved to satisfy a translation inclusion.

Theorem 8 For any $\mathrm{u}, v \in \mathrm{U}$ and $\mathrm{A} \subseteq \mathrm{X}$, we have

$$
f(u, f(v, A)) \subseteq f(u+v, A) .
$$


Proof. By Definition 4 and the assumed superadditivity property of $\alpha$, we have

$$
\begin{aligned}
& f(u, f(v, A))=\alpha(u)[f(v, A)]=\alpha(u)[\alpha(v)[A]] \\
& =(\alpha(u) \circ \alpha(v))[A] \subseteq \alpha(u+v)[A]=f(u+v, A) .
\end{aligned}
$$

Remark 10 Thus, by identifying singleton with their elements, we may also write

$$
f(u, f(v, x)) \subseteq f(u+v, x)
$$

for all $u, v \in U$ and $x \in X$.

Now, to illustrate the appropriateness of Definition 4, we can also state

Example 1 If in particular $\alpha$ is as in Definition 3, then by Definition 4 we have

$$
f(n, A)=\alpha(n)[A]=\varphi^{n}[A]
$$

for all $\mathrm{n} \in \overline{\mathbb{N}}_{0}$ and $\mathrm{A} \subseteq \mathrm{X}$. Thus, in particular $\mathrm{f}(\mathrm{O}, \mathrm{A})=\mathrm{A}$ for all $\mathrm{A} \subseteq \mathrm{X}$.

\section{From a translation inclusion to a Sincov inclusion}

Now, as an extension of our former observations, we may also naturally start with the following

Notation 3 Suppose that $\mathrm{U}$ is an additive groupoid, $\mathrm{X}$ is a goset and $\mathrm{f}$ is a function of $\mathrm{U} \times \mathrm{X}$ to $\mathrm{X}$ such that $\mathrm{f}$ is increasing in its second variable and

$$
f(u, f(v, x)) \leq f(u+v, x)
$$

for all $\mathrm{u}, v \in \mathrm{U}$ and $\mathrm{x} \in \mathrm{X}$.

Thus, improving an idea of Frege $[15,16]$, we may also naturally introduce

Definition 5 For any $x, y \in X$, define

$$
F(x, y)=\{u \in U: \quad x \leq f(u, y)\} .
$$

Thus, $\mathrm{F}$ may be considered as a relation on $\mathrm{X}^{2}$ to $\mathrm{U}$, or as a function of $\mathrm{X}^{2}$ to $\mathcal{P}(\mathrm{U})$, which can be proved to satisfy a Sincov type inclusion. 
Theorem 9 For any $x, y, z \in X$, we have

$$
\mathrm{F}(x, y)+\mathrm{F}(\mathrm{y}, z) \subseteq \mathrm{F}(x, z) .
$$

Proof. If

$$
u \in F(x, y) \quad \text { and } \quad v \in F(y, z) \text {, }
$$

then by Definition 5 we get

$$
x \leq f(u, y) \quad \text { and } \quad y \leq f(v, z) .
$$

Hence, by using the assumed increasingness and translation property of $f$, we can infer that

$$
x \leq f(u, y) \leq f(u, f(v, z)) \leq f(u+v, z) .
$$

Therefore, by Definition 5, we have

$$
u+v \in F(x, z) \text {. }
$$

Thus, the required inclusion is true.

Now, to illustrate the appropriateness of Definition 5, we can also state

Example 2 If $\mathrm{f}$ is as in Example 1, then by Definition 5 we have

$$
\mathrm{F}(\mathrm{A}, \mathrm{B})=\left\{\mathrm{n} \in \overline{\mathbb{N}}_{0}: \quad \mathrm{A} \subseteq \mathrm{f}(\mathrm{n}, \mathrm{B})\right\}=\left\{\mathrm{n} \in \overline{\mathbb{N}}_{0}: \quad A \subseteq \varphi^{\mathrm{n}}[\mathrm{B}]\right\}
$$

for all $\mathrm{A}, \mathrm{B} \subseteq \mathrm{X}$. Thus, in particular $\mathrm{O} \in \mathrm{F}(\mathrm{A}, \mathrm{A})$ for all $\mathrm{A} \subseteq \mathrm{X}$.

Remark 11 By Aczél [1, pp. 223, 303 and 353], Sincov's functional equation and its generalizations have been investigated by a surprisingly great number of authors.

For some more recent investigations, see [4, 33, 38, 27, 34, 35, 7, 8, 3, 14, 13]. The most relevant ones are the set-valued considerations of Smajdor [38] and Augustová and Klapka [3].

Moreover, it is noteworthy that, by using the famous partial operation

$$
(x, y) \bullet(y, z)=(x, z)
$$

the above Sincov inclusion can be turned into a restricted Cauchy inclusion.

Therefore, some of the methods of the theory of superadditive functions and relations $[28,17,29,19]$ can certainly be applied to investigate the corresponding Sincov inequalities and inclusions. 


\section{Some immediate consequences of a Sincov inclu- sion}

Now, motivated by our former observations, we may also naturally introduce the following notations and definitions.

Notation 4 In what follows, we shall also assume that $\mathrm{X}$ is a set and $\mathrm{U}$ is an additive groupoid. Moreover, we shall suppose that $\mathrm{F}$ is a relation on $\mathrm{X}^{2}$ to $\mathrm{U}$.

Definition 6 The relation $F$ will be called supertriangular if

$$
F(x, y)+F(y, z) \subseteq F(x, z)
$$

for all $x, y, z \in X$.

Remark 12 Now, the relation F may also be naturally called subtriangular if the reverse inclusion holds. Moreover, F may be naturally called triangular if it is both subtriangular and supertriangular.

Subtriangular relations are certainly more important than the supertriangular ones. Namely, if a function $\mathrm{d}$ of $\mathrm{X}^{2}$ to $[0,+\infty]$ satisfies the triangle inequality

$$
d(x, z) \leq d(x, y)+d(y, z)
$$

for all $x, y, z \in X$, then the relation $F$, defined such that

$$
F(x, y)=[0, d(x, y)] \quad(F(x, y)=[-d(x, y), d(x, y)])
$$

for all $x, y \in X$, can, in general, be proved to be only subtriangular [2].

The $y=x, y=z$ and $z=x$ particular cases of the inclusion considered in Definition 6 strongly suggest the introduction of the following

Definition 7 For any $x, y \in X$, define

$$
R(x, y)=F(y, x) \quad \text { and } \quad S(x, y)=F(x, y)+R(x, y) .
$$

Moreover, for any $x \in X$, define

$$
\Phi(x)=F(x, x) \quad \text { and } \quad \Psi(x)=\bigcup_{y \in X} S(x, y) .
$$

Thus, $\mathrm{R}$ and $\mathrm{S}$ may be considered as relations on $\mathrm{X}^{2}$ to $\mathrm{U}$, and $\Phi$ and $\Psi$ may be considered as relations on $\mathrm{X}$ to $\mathrm{U}$.

Concerning these relations, we can easily prove the following 
Theorem 10 For any $\mathrm{x}, \mathrm{y} \in \mathrm{X}$ we have

(1) $\Phi(x)+\Phi(x) \subseteq \Psi(x)$;

(2) $\mathrm{R}(\mathrm{x}, \mathrm{x})=\Phi(\mathrm{x})$;

(3) $S(x, x)=\Phi(x)+\Phi(x)$.

Proof. By Definition 7, we evidently have

$$
R(x, x)=F(x, x)=\Phi(x),
$$

and thus also

$$
S(x, x)=F(x, x)+R(x, x)=\Phi(x)+\Phi(x) .
$$

Hence, by using the definition of $\Psi$, we can also easily note that

$$
\Phi(x)+\Phi(x)=S(x, x) \subseteq \bigcup_{y \in X} S(x, y)=\Psi(x) .
$$

Therefore, assertions (2), (3) and (1) are true.

Now, as a counterpart of [38, Lemma 1] of Wilhelmina Smajdor, we can also prove the following

Theorem 11 If $\mathrm{F}$ is supertriangular, then for any $\mathrm{x}, \mathrm{y} \in \mathrm{X}$ we have

(1) $\Psi(x) \subseteq \Phi(x)$;

(2) $\Phi(x)+F(x, y) \subseteq F(x, y)$;

(3) $F(x, y)+\Phi(y) \subseteq F(x, y)$.

Proof. By using Definition 7 and the corresponding particular cases of the inclusion considered in Definition 6, we can easily see that

$$
\Phi(x)+F(x, y)=F(x, x)+F(x, y) \subseteq F(x, y)
$$

and

$$
F(x, y)+\Phi(y)=F(x, y)+F(y, y) \subseteq F(x, y)
$$

Moreover,

$$
S(x, y)=F(x, y)+R(x, y)=F(x, y)+F(y, x) \subseteq F(x, x)=\Phi(x),
$$

and thus also

$$
\Psi(x)=\bigcup_{y \in X} S(x, y) \subseteq \bigcup_{y \in X} \Phi(x) \subseteq \Phi(x) .
$$

Therefore, assertions (2), (3) and (1) are true even if only some consequences of the assumed inclusion property of $\mathrm{F}$ are supposed to hold.

Now, as an immediate consequence of the above two theorems, we can also state 
Corollary 2 If $\mathrm{F}$ is supertriangular, then for any $\mathrm{x}, \mathrm{y} \in \mathrm{X}$ we have
(1) $\Phi(x)+\Phi(x) \subseteq \Phi(x)$;
(2) $\Psi(x)+\Psi(x) \subseteq \Psi(x)$;
(3) $\Psi(x)+\Phi(x) \subseteq \Psi(x)$;
(4) $\Phi(x)+\Psi(x) \subseteq \Psi(x)$;
(5) $\Psi(x)+F(x, y) \subseteq F(x, y)$;
(6) $F(x, y)+\Psi(y) \subseteq F(x, y)$.

Remark 13 By [8], in addition to Definition 6, the separability equation

$$
\mathrm{F}(x, y)+F(y, z)=F(x, z)+\Phi(y)
$$

may also be naturally investigated.

Moreover, if in particular $\mathrm{U}$ is a group, then in addition to Definition 7 , the disymmetry relation $\mathrm{D}$ of $\mathrm{F}$, defined such that $\mathrm{D}(\mathrm{x}, \mathrm{y})=\mathrm{F}(\mathrm{x}, \mathrm{y})-\mathrm{R}(\mathrm{x}, \mathrm{y})$ for all $x, y \in X$, may also be naturally investigated.

\section{The particular case when $U$ has a zero element}

Theorem 12 If $\mathrm{F}$ is supertriangular, $\mathrm{U}$ has a one-sided zero element $\mathrm{O}$ and $x \in X$ is such that $0 \in \Phi(x)$, then
(1) $\Phi(x)=\Psi(x)$;
(2) $\Phi(x)=\Phi(x)+\Phi(x)$.

Proof. If 0 is a right zero element of $\mathrm{U}$, then by using Theorems 10 and 11 we can see that

$$
\Phi(x)=\Phi(x)+\{0\} \subseteq \Phi(x)+\Phi(x) \subseteq \Psi(x) \subseteq \Phi(x) .
$$

While, if 0 is a left zero element of $\mathrm{U}$, then we can quite similarly see that

$$
\Phi(x)=\{0\}+\Phi(x) \subseteq \Phi(x)+\Phi(x) \subseteq \Psi(x) \subseteq \Phi(x) .
$$

Therefore, in both cases, the required equalities are true.

Remark 14 Note that if in particular $F$ is as in Example 2, then $0 \in \Phi(A)$ holds for all $A \subseteq X$. Therefore, the above theorem can be applied.

Now, by using a somewhat more complicated argument, we can also prove

Theorem 13 If $\mathrm{F}$ is supertriangular, $\mathrm{U}$ has a one-sided zero element $\mathrm{O}$ and $\mathrm{x}, \mathrm{y} \in \mathrm{X}$ are such that

then

$$
0 \in F(x, y) \cap F(y, x)
$$

(1) $\Phi(x)=\Psi(x)=F(x, y)=S(x, y)$;

(2) $\Phi(x)=\Phi(x)+\Phi(y)$. 
Proof. If 0 is a right zero element of $\mathrm{U}$, then by using Theorem 11 we can see that

$$
\begin{aligned}
\Phi(x) & =\Phi(x)+\{0\} \subseteq \Phi(x)+F(x, y) \subseteq F(x, y)=F(x, y)+\{0\} \\
& \subseteq F(x, y)+F(y, x)=F(x, y)+R(x, y)=S(x, y) \subseteq \Psi(x) \subseteq \Phi(x) .
\end{aligned}
$$

While, if 0 is a left zero element of $\mathrm{U}$, then we can quite similarly obtain

$$
\begin{aligned}
\Phi(x) & =\{0\}+\Phi(x) \subseteq F(y, x)+\Phi(x) \subseteq F(y, x)=\{0\}+F(y, x) \\
& \subseteq F(x, y)+F(y, x)=F(x, y)+R(x, y)=S(x, y) \subseteq \Psi(x) \subseteq \Phi(x) .
\end{aligned}
$$

Therefore, in both cases, assertion (1) is true.

Now, assertion (2) can be easily derived from assertion (1), by noticing that

$$
\Phi(x)=S(x, y)=F(x, y)+R(x, y)=F(x, y)+F(y, x)=\Phi(x)+\Phi(y) .
$$

From this theorem, it is clear that in particular we also have the following

Corollary 3 If $\mathrm{F}$ is supertriangular and $\mathrm{U}$ has a one-sided zero element 0 such that $0 \in \mathrm{F}(\mathrm{x}, \mathrm{y})$ for all $\mathrm{x}, \mathrm{y} \in \mathrm{X}$, then for any $\mathrm{x}, \mathrm{y} \in \mathrm{X}$ we have
(1) $\Phi(x)=\Psi(x)=F(x, y)=S(x, y)$;
(2) $\Phi(x)=\Phi(x)+\Phi(y)$

\section{The particular case when $U$ is a group}

By using an argument of Frege $[15,16]$ and Sincov [36, 23], we can prove

Theorem 14 If $\mathrm{F}$ is a nonpartial, triangular function and $\mathrm{U}$ is a group, then there exists a function $\xi$ of $\mathrm{X}$ to $\mathrm{U}$ such that

$$
F(x, y)=\xi(x)-\xi(y)
$$

for all $\mathrm{x}, \mathrm{y} \in \mathrm{X}$.

Proof. By choosing $z \in X$, and defining

$$
\xi(x)=F(x, z)
$$

for all $x \in X$, we can see that

$$
F(x, y)+\xi(y)=F(x, y)+F(y, z)=F(x, z)=\xi(x),
$$

and thus $F(x, y)=\xi(x)-\xi(y)$ for all $x, y \in X$. 
Remark 15 If $F$ is nonpartial and supertriangular and $\mathrm{U}$ is a group, then by using a similar argument we can only prove that

$$
\mathrm{F}(x, y) \subseteq \bigcap_{z \in X}(\mathrm{~F}(x, z)-\mathrm{F}(y, z))
$$

for all $x, y \in X$.

Now, analogously to [38, Theorem 1] of Wilhelmina Smajdor, we can also prove

Theorem 15 If $\mathrm{F}$ is nonpartial and supertriangular, $\mathrm{U}$ is a commutative group and $\phi$ is a triangular selection function of $\mathrm{F}$, then

$$
\mathrm{F}(x, y)=\phi(x, y)+\Phi(x)
$$

for all $\mathrm{x}, \mathrm{y} \in \mathrm{X}$.

Proof. Define

$$
G(x, y)=-\phi(x, y)+F(x, y)
$$

for all $x, y \in X$.

Then, because of $\phi(x, y) \in F(x, y)$, we evidently have

$$
0=-\phi(x, y)+\phi(x, y) \in-\phi(x, y)+F(x, y)=G(x, y)
$$

for all $x, y \in X$. Moreover, by using the assumed triangularity properties of $\phi$ and $F$, we can easily see that

$$
\begin{aligned}
& \mathrm{G}(x, y)+\mathrm{G}(y, z)=-\phi(x, y)+F(x, y)-\phi(y, z)+F(y, z)= \\
& \quad-(\phi(x, y)+\phi(y, z))+F(x, y)+F(y, z) \subseteq-\phi(x, z)+F(x, z)=G(x, z)
\end{aligned}
$$

for all $x, y, z \in X$.

Hence, by using Corollary 3 and the simple observation that

$$
\phi(x, x)+\phi(x, x)=\phi(x, x),
$$

and thus $\phi(x, x)=0$ for all $x \in X$, we can already infer that

$$
\mathrm{G}(x, y)=\mathrm{G}(x, x)=-\phi(x, x)+\mathrm{F}(x, x)=\Phi(x),
$$

and thus

$$
-\phi(x, y)+F(x, y)=\Phi(x)
$$

for all $x, y \in X$. Therefore, the required equality is also true. 
Remark 16 It can be easily seen that a converse of Theorem 14 is also true. Therefore, if $\mathrm{F}$ is nonpartial and $\mathrm{U}$ is a group, then to find a triangular selection function $\phi$ of $F$, it is enough to find only a function $\xi$ of $X$ to $U$ such that

$$
\xi(x)-\xi(y) \in F(x, y)
$$

for all $x, y \in X$.

\section{The particular case when $U$ is a commutative groupoid}

Theorem 16 If $\mathrm{F}$ is supertriangular and $\mathrm{U}$ is commutative, then $\mathrm{R}$ is also supertriangular.

Proof. By Definitions 6 and 7 and the commutativity of $\mathrm{U}$, we have

$$
\begin{aligned}
R(x, y)+R(y, z) & =F(y, x)+F(z, y) \\
& =F(z, y)+F(y, x) \subseteq F(z, x)=R(x, z)
\end{aligned}
$$

for all $x, y, z \in X$.

Theorem 17 If $\mathrm{U}$ is commutative, then for any $\mathrm{x}, \mathrm{y}, z \in \mathrm{X}$ we have

(1) $S(x, y)=S(y, x)$;

(2) $S(x, y) \subseteq \Psi(x) \cap \Psi(y)$.

Proof. By Definition 7 and the commutativity of $\mathrm{U}$, we have

$$
\begin{aligned}
S(x, y) & =F(x, y)+R(x, y)=R(y, x)+F(y, x) \\
& =F(y, x)+R(y, x)=S(y, x) .
\end{aligned}
$$

Moreover, by the definition of $\Psi$, it is clear that $S(x, y) \subseteq \Psi(x)$. Hence, by using the above symmetry property of $S$, we can already infer that

$$
S(x, y)=S(y, x) \subseteq \Psi(y),
$$

and thus $S(x, y) \subseteq \Psi(x) \cap \Psi(y)$ also holds.

Remark 17 Thus, if $\mathrm{U}$ is commutative, then $\mathrm{S}$ is already pointwise symmetric in the sense that $S(x, y)=S(y, x)$ for all $x, y \in X$.

Now, concerning the relation $S$, we can also prove the following 
Theorem 18 If $\mathrm{F}$ is supertriangular and $\mathrm{U}$ is a commutative semigroup, then $\mathrm{S}$ is also supertriangular.

Proof. By using Definition 7, Theorem 16 and the commutativity and associativity of $\mathrm{U}$, we can see that

$$
\begin{aligned}
S(x, y)+S(y, z)= & F(x, y)+R(x, y)+F(y, z)+R(y, z) \\
= & F(x, y)+F(y, z)+R(x, y)+R(y, z) \\
& \subseteq F(x, z)+R(x, z)=S(x, z)
\end{aligned}
$$

for all $x, y, z \in X$.

\section{The particular case when $F$ is pointwise symmet- ric}

In addition to Theorem 17, we can also prove the following

Theorem 19 If $x, y \in X$ such that $\mathrm{F}(x, y)=\mathrm{F}(\mathrm{y}, \mathrm{x})$, then

(1) $\mathrm{R}(x, y)=\mathrm{F}(x, y)$;

(2) $S(x, y)=S(y, x)$;

(3) $S(x, y)=F(x, y)+F(x, y)$;

(4) $2 \mathrm{~F}(x, y) \subseteq S(x, y) \subseteq \Psi(x) \cap \Psi(y)$.

Proof. By Definition 7 and the assumed symmetry property of $F$, we have

$$
R(x, y)=F(y, x)=F(x, y)
$$

and thus also

$$
S(x, y)=F(x, y)+R(x, y)=F(x, y)+F(x, y) .
$$

Thus, assertions (1) and (3) are true.

Now, we can also easily see that

$$
S(y, x)=F(y, x)+F(y, x)=F(x, y)+F(x, y)=S(x, y) .
$$

Therefore, assertion (2) is also true.

Hence, as in the proof of Theorem 17, we can already infer that

$$
S(x, y) \subseteq \Psi(x) \cap \Psi(y) .
$$


Therefore, to complete the proof of assertion (4), it remains to note only that now

$$
2 F(x, y) \subseteq F(x, y)+F(x, y)=S(x, y)
$$

is also true.

Remark 18 Thus, not only the commutativity of $\mathrm{U}$, but the pointwise symmetry of $F$ also implies the pointwise symmetry of $S$.

By [8], in addition to the pointwise symmetry of $F$, one may also naturally investigate the case when $\mathrm{F}$ is only weightable in the sense that

$$
w(x)+F(x, y)=R(x, y)+w(y)
$$

for all $x, y \in X$ and some function (or relation) $w$ on $X$ to $\mathrm{U}$.

However, it is now more important to note that, as an immediate consequence of our former results, we can also state

Corollary 4 If $\mathrm{F}$ is supertriangular and $\mathrm{U}$ is commutative, then for any $x, y \in X$ we have

$$
2 S(x, y) \subseteq S(x, y)+S(y, x) \subseteq S(x, x) \cap S(y, y) .
$$

Remark 19 Note that the latter corollary only needs the important consequence of the assumed inclusion property of $F$ that $F(x, y)+F(y, x) \subseteq F(x, x)$ for all $x, y \in X$.

In Theorem 11, by using Definition 7 , the latter property has been reformulated in the shorter form that $\Psi(x) \subseteq \Phi(x)$ for all $x \in X$. Now, this already implies that $\Psi$ is a selection relation of $\Phi$. Namely, if $x \in X$ such that $\Phi(x) \neq \emptyset$, then because of $\Phi(x)+\Phi(x) \subseteq \Psi(x)$, we also have $\Psi(x) \neq \emptyset$.

\section{The particular case when $U$ is a group and $F$ is pointwise skew symmetric}

Analogously to Theorem 19, we can also prove the following

Theorem 20 If $\mathrm{U}$ is a group and $\mathrm{x}, \mathrm{y} \in \mathrm{X}$ such that $\mathrm{F}(\mathrm{x}, \mathrm{y})=-\mathrm{F}(\mathrm{y}, \mathrm{x})$, then

(1) $R(x, y)=-F(x, y)$

(2) $S(x, y)=-S(y, x)$;

(3) $S(x, y)=F(x, y)-F(x, y)$;

(4) $S(x, y) \subseteq \Psi(x) \cap(-\Psi(y))$. 
Proof. To prove (4), note that now, in addition to $S(x, y) \subseteq \Psi(x)$, we also have

$$
S(x, y)=-S(y, x) \subseteq-\Psi(y),
$$

and thus $S(x, y) \subseteq \Psi(x) \cap(-\Psi(y))$ also holds.

Remark 20 If in addition to the assumptions of this theorem $F(x, y) \neq \emptyset$ also holds, then from assertion (3) we can infer that $0 \in S(x, y)$.

Now, by using the corresponding definitions and Theorem 20, we can also prove

Theorem 21 If $\mathrm{U}$ is a group and $\mathrm{F}$ is pointwise skew symmetric, then for any $x \in X$ we have
(1) $\Phi(x)=-\Phi(x)$;
(2) $\Psi(x)=-\Psi(x)$.

Proof. To prove (2), note that by Definition 7 and Theorem 20 we have

$$
\Psi(x)=\bigcup_{y \in X} S(x, y)=\bigcup_{y \in X}(-S(x, y))=-\bigcup_{y \in X} S(x, y)=-\Psi(x)
$$

for all $x \in X$.

Remark 21 If in addition to the assumptions of this theorem, $\Phi(x) \neq \emptyset$ also holds, then from the inclusion

$$
\Phi(x)-\Phi(x)=\Phi(x)+\Phi(x) \subseteq \Psi(x),
$$

we can infer that $0 \in \Psi(x)$. Therefore, if in addition $F$ is supertriangular, then because Theorem 11, we also have $0 \in \Phi(x)$.

Thus, by Theorem 12, we can also state the following

Theorem 22 If $\mathrm{U}$ is a group and $\mathrm{F}$ is nonpartial, supertriangular and pointwise skew symmetric, then for any $\mathrm{x} \in \mathrm{X}$ we have
(1) $\Phi(x)=\Psi(x)$;
(2) $\Phi(x)=\Phi(x)+\Phi(x)$.

Now, by Theorems 20 and 21, we can also state the following

Theorem 23 If $\mathrm{U}$ is a group and $\mathrm{F}$ is a nonpartial, pointwise skew symmetric function, then for any $\mathrm{x}, \mathrm{y} \in \mathrm{X}$ we have
(1) $S(x, y)=0$;
(2) $\Phi(x)=\Psi(x)=0$. 
The following example shows the three important consequences of the inclusion considered in Definition 6 do not imply, even in a very simple case, the validity of this inclusion itself.

Example 3 If

$$
F(x, y)=\operatorname{sgn}(x-y)
$$

for all $\mathrm{x}, \mathrm{y} \in \mathbb{R}$, then $\mathrm{F}$ is a skew symmetric function of $\mathbb{R}^{2}$ to $\mathbb{R}$ such that, under the notation $\Phi(\mathrm{x})=\mathrm{F}(\mathrm{x}, \mathrm{x})$, for any $\mathrm{x}, \mathrm{y} \in \mathrm{X}$ we have

(1) $F(x, y)+F(y, x)=\Phi(x)$;

(2) $\Phi(x)+F(x, y)=F(x, y) ; \quad(3) \quad F(x, y)+\Phi(y)=F(x, y)$.

However, $\mathrm{F}$ is not either supertriangular nor subtriangular in both functional and relational sense.

Namely, for instance, we have

$$
\mathrm{F}(2,1)+\mathrm{F}(1,0)=2 \quad \text { and } \quad \mathrm{F}(2,0)=1,
$$

and

$$
\mathrm{F}(0,1)+\mathrm{F}(1,2)=-2 \quad \text { and } \quad \mathrm{F}(0,2)=-1 .
$$

\section{The particular case when $U$ is cancellative}

Definition 8 In what follows, we shall denote by lcan $(U)$ and rcan(U) the family of all left-cancellable and right-cancellable elements of the groupoid $\mathrm{U}$, respectively.

Moreover, we shall also write $\operatorname{can}(\mathrm{U})=\operatorname{lcan}(\mathrm{U}) \cap \operatorname{rcan}(\mathrm{U})$.

Remark 22 Thus, for any $u \in U$, we have $u \in \operatorname{lcan}(\mathrm{U})$ if and only if $u+v=$ $u+w$ implies $u=w$ for all $v, w \in U$.

Moreover, for instance, we can state that $\mathrm{U}$ is left-cancellative if and only if $\operatorname{lcan}(\mathrm{U})=\mathrm{U}$.

Lemma 1 For any $\mathrm{V}, \mathrm{W} \subseteq \mathrm{U}$,

(1) $\operatorname{card}(\mathrm{V}+\mathrm{W}) \leq 1$ and $\mathrm{V} \cap \operatorname{lcan}(\mathrm{U}) \neq \emptyset$ imply that $\operatorname{card}(\mathrm{W}) \leq 1$;

(2) $\operatorname{card}(\mathrm{V}+\mathrm{W}) \leq 1$ and $\mathrm{W} \cap \operatorname{rcan}(\mathrm{U}) \neq \emptyset$ imply that $\operatorname{card}(\mathrm{V}) \leq 1$.

Proof. Assume that the conditions of (1) hold, $v \in \mathrm{V} \cap \operatorname{lcan}(\mathrm{U})$ and $w_{1}, w_{2} \in$ $\mathrm{W}$. Then, we have $v+w_{1}, v+w_{2} \in \mathrm{V}+\mathrm{W}$. Hence, by using that card $(\mathrm{V}+\mathrm{W}) \leq$ 1 , we can infer that $v+w_{1}=v+w_{2}$. Moreover, since $v \in \operatorname{lcan}(\mathrm{U})$, we can also state that $w_{1}=w_{2}$. Therefore, $\operatorname{card}(W) \leq 1$, and thus (1) also holds. 
The proof of assertion (2) is quite similar.

Now, by using this lemma, we can give some reasonable sufficient conditions in order that a suppertriangular relation should be a function.

Theorem 24 If $\mathrm{F}$ is supertriangular and there exist $\mathrm{x}_{0}, \mathrm{y}_{0} \in \mathrm{X}$ such that

(1) $\operatorname{card}\left(\mathrm{F}\left(\mathrm{x}_{0}, \mathrm{y}_{0}\right)\right) \leq 1$;

(2) $\mathrm{F}\left(x, y_{0}\right) \cap \operatorname{rcan}(\mathrm{U}) \neq \emptyset$ for all $x \in X$;

(3) $\mathrm{F}\left(\mathrm{x}_{0}, \mathrm{y}\right) \cap \operatorname{lcan}(\mathrm{U}) \neq \emptyset$ for all $\mathrm{y} \in \mathrm{X}$;

then $\operatorname{card}(\mathrm{F}(\mathrm{x}, \mathrm{y})) \leq 1$ for all $\mathrm{x}, \mathrm{y} \in \mathrm{X}$, and thus $\mathrm{F}$ is a function.

Proof. By the assumed inclusion property of $F$, we have

$$
F\left(x_{0}, x\right)+F\left(x, y_{0}\right) \subseteq F\left(x_{0}, y_{0}\right)
$$

for all $x \in X$. Hence, by using conditions (1) and (3) and Lemma 1, we can infer that

(a) $\operatorname{card}\left(F\left(x, y_{0}\right)\right) \leq 1$ for all $x \in X$.

Now, by the assumed inclusion property of $F$, we also have

$$
F(x, y)+F\left(y, y_{0}\right) \subseteq F\left(x, y_{0}\right)
$$

for all $x, y \in X$. Hence, by using assertion (a) condition (2) and Lemma 1, we can infer that

(b) $\operatorname{card}(F(x, y)) \leq 1$ for all $x, y \in X$.

Thus, the required assertion is true.

From this theorem, by using Theorem 14, we can immediately derive

Corollary 5 If $\mathrm{F}$ is nonpartial and supertriangular, $\mathrm{U}$ is a group and $\operatorname{card}\left(\mathrm{F}\left(\mathrm{x}_{0}, \mathrm{y}_{0}\right)\right)=1$ for some $\mathrm{x}_{0}, \mathrm{y}_{0} \in \mathrm{X}$, then there exists a function $\xi$ of $\mathrm{X}$ to $\mathrm{U}$ such that

$$
F(x, y)=\xi(x)-\xi(y)
$$

for all $\mathrm{x}, \mathrm{y} \in \mathrm{X}$.

\section{The particular case when $U$ has a suitable dis- tance function}

Remark 23 A function d of $X^{2}$ to $[0,+\infty]$ is usually called a distance function on $X$. 
Moreover, the extended real number

$$
d(X)=\operatorname{diam}(X)=\sup \{d(x, y): \quad x, y \in X\}
$$

is called the diameter of $\mathrm{X}$.

Remark 24 Thus, we have $d(X)=-\infty$ if $X=\emptyset$, and $d(X) \geq 0$ if $X \neq \emptyset$. Moreover, if $X \neq \emptyset$, then $\operatorname{card}(X)=+\infty$ may also hold even if $X$ is finite.

Definition 9 A distance function $\mathrm{d}$ on $\mathrm{X}$ will be called admissible if

(a) $\mathrm{d}(\mathrm{X})<+\infty$;

(b) $d(x, y)=0$ implies $x=y$ for all $x, y \in X$.

Moreover, the distance function $\mathrm{d}$ will be called extremal if

(c) for any $x, y \in X$ there exist $c \in] 1,+\infty[$ and $z, w \in X$ such that

$$
\operatorname{cd}(x, y) \leq d(z, w)
$$

Remark 25 If $X$ is an additive groupoid, then to satisfy condition (c) we may naturally assume that for any $x, y \in X$, there exists $n \in \mathbb{N} \backslash\{1\}$ such that

$$
n d(x, y) \leq d(n x, n y) .
$$

Namely, if $\mathrm{X}$ is a commutative abelian group and $\mathrm{p}$ is a function of $\mathrm{U}$ to $[0,+\infty]$ such that

$$
n p(x) \leq p(n x)
$$

for all $n \in \mathbb{N}$ and $x \in X$, then by defining

$$
d(x, y)=p(-x+y)
$$

for all $x, y \in X$, we have

$$
\begin{aligned}
n d(x, y) & =n p(-x+y) \leq p(n(-x+y)) \\
& =p(n(-x)+n y)=p(-n x+n y)=d(n x, n y)
\end{aligned}
$$

for all $\mathrm{n} \in \mathbb{N}$ and $x, y \in \mathrm{U}$.

The introduction of Definition 9 can only be motivated by the following

Lemma 2 If there exists an extremal, admissible distance function $\mathrm{d}$ on $\mathrm{X}$, then $\operatorname{card}(\mathrm{X}) \leq 1$. 
Proof. If $X=\emptyset$, then the required assertion trivially holds. Therefore, we may assume that $X \neq \emptyset$, and thus $d(X) \neq-\infty$. Now, by condition (a), we can state that $d(X) \in \mathbb{R}$. Moreover, since $d$ is nonnegative, we can now also note that $\mathrm{d}(\mathrm{X}) \geq 0$.

Thus, for every $\varepsilon>0$, we have

$$
d(X)-\varepsilon<d(X) .
$$

Therefore, by the definition of $d(X)$, there exist $x, y \in X$ such that $d(X)-\varepsilon<d(x, y)$, and thus

$$
\mathrm{d}(X)<\mathrm{d}(x, y)+\varepsilon .
$$

Moreover, by condition (c), there exist $\mathrm{c} \in] 1,+\infty[$ and and $z, w \in X$ such that

$$
\operatorname{cd}(x y) \leq \mathrm{d}(z, w) .
$$

Combining the above two inequalities, we can see that

$$
\operatorname{cd}(x, y)<d(z, w) \leq d(X)<d(x, y)+\varepsilon,
$$

and thus $(c-1) d(x, y)<\varepsilon$. Hence, by letting $\varepsilon$ tend to zero, we can infer that $(c-1) d(x, y) \leq 0$. Therefore, since $c-1>0$, we necessarily have $d(x, y) \leq 0$, and hence $d(x, y) \leq 0$ by the nonnegativity of $d$. Thus, we actually have

$$
\mathrm{d}(X)<\mathrm{d}(x, x)+\varepsilon=\varepsilon .
$$

Hence, by letting $\varepsilon$ tend to zero, we can infer that $d(X) \leq 0$, and thus also $\mathrm{d}(\mathrm{X})=0$ by the nonnegativity of $\mathrm{d}(\mathrm{X})$.

This, by condition (b), already implies that $\operatorname{card}(X)=1$. Namely, if this is not the case, then by the assumption $X \neq \emptyset$, there exist $x, y \in X$ such that $x \neq y$. Hence, by condition (b) and the nonnegativity of $d$, we can infer that $d(x, y)>0$, and thus also $d(X)>0$ by the definition of $d(X)$. This contradiction proves that $\operatorname{card}(\mathrm{X})=1$.

Remark 26 From condition (c), by induction, we can infer that there exist sequences $\left(c_{n}\right)_{n=1}^{\infty}$ in $] 1,+\infty\left[\right.$ and $\left(x_{n}\right)_{n=1}^{\infty}$ and $\left(y_{n}\right)_{n=1}^{\infty}$ in $X$ such that

$$
d(x, y) \prod_{i=0}^{n} c_{i} \leq d\left(x_{n}, y_{n}\right)
$$

for all $n \in \mathbb{N}$. However, this fact cannot certainly be used to give a simpler proof for Lemma 2. 
From Theorem 24, by using Lemma 2, we can immediately derive

Theorem 25 If $\mathrm{F}$ is supertriangular and there exist $\mathrm{x}_{0}, \mathrm{y}_{0} \in \mathrm{X}$, such that

(1) $\mathrm{F}\left(x, y_{0}\right) \cap \operatorname{rcan}(\mathrm{U}) \neq \emptyset$; for all $\mathrm{x} \in \mathrm{X}$;

(2) $\mathrm{F}\left(\mathrm{x}_{0}, \mathrm{y}\right) \cap \mathrm{lcan}(\mathrm{U}) \neq \emptyset$; for all $\mathrm{y} \in \mathrm{X}$;

(3) there exists an extremal, admissible distance function on $\mathrm{F}\left(\mathrm{x}_{0}, \mathrm{y}_{0}\right)$; then $\operatorname{card}(\mathrm{F}(\mathrm{x}, \mathrm{y})) \leq 1$ for all $\mathrm{x}, \mathrm{y} \in \mathrm{X}$, and thus $\mathrm{F}$ is a function.

Proof. By assumption (3) and Lemma 2, we have $\operatorname{card}\left(\mathrm{F}\left(\mathrm{x}_{0}, \mathrm{y}_{0}\right)\right) \leq 1$. Hence, by Theorem 24, we can see that the required assertion is also true.

\section{Contructions of supertriangular relations}

Theorem 26 If $\mathrm{V}$ is a subgroupoid of $\mathrm{U}$ and

$$
\mathrm{F}(\mathrm{x}, \mathrm{y})=\mathrm{V}
$$

for all $\mathrm{x}, \mathrm{y} \in \mathrm{X}$, then $\mathrm{F}$ is a supertriangular relation on $\mathrm{X}$ to $\mathrm{U}$.

Proof. We evidently have

$$
\mathrm{F}(x, y)+\mathrm{F}(\mathrm{y}, z)=\mathrm{V}+\mathrm{V} \subseteq \mathrm{V}=\mathrm{F}(\mathrm{x}, z)
$$

for all $x, y, z \in X$.

Remark 27 Conversely, note that if $F$ is a supertriangular relation on $X^{2}$ to $\mathrm{U}$, then by Corollary $2 \Phi(\mathrm{x})=\mathrm{F}(\mathrm{x}, \mathrm{x})$ is a subgroupoid of $\mathrm{U}$ for all $\mathrm{x} \in \mathrm{X}$.

Now, as a converse to Theorem 14, we can also easily prove the following

Theorem 27 If $\xi$ is a function of $\mathrm{X}$ to $\mathrm{U}, \mathrm{U}$ is a group and

$$
\mathrm{F}(x, y)=\xi(x)-\xi(y)
$$

for all $\mathrm{x}, \mathrm{y} \in \mathrm{X}$, then $\mathrm{F}$ is a triangular function of $\mathrm{X}^{2}$ to $\mathrm{U}$.

Proof. We evidently have

$$
F(x, y)+F(y, z)=\xi(x)-\xi(y)+\xi(y)-\xi(z)=\xi(x)-\xi(z)=F(x, z)
$$

for all $x, y, z \in X$. 
Remark 28 If $\xi$ is only a relation of $X$ to $U, U$ is a group and $F(x, y)=$ $\xi(x)-\xi(y)$ for all $x, y \in X$, then by using a similar argument we can only prove that $\mathrm{F}$ is a subtriangular relation of $\mathrm{X}^{2}$ to $\mathrm{U}$.

In addition to the above two theorems, it is also worth proving that the family of all supertriangular relations is closed under the usual pointwise operations.

Theorem 28 If $\mathrm{F}$ is a supertriangular relation on $\mathrm{X}^{2}$ to $\mathrm{U}$ and $\mathrm{U}$ is a commutative semigroup, then $\mathrm{nF}$ is also a supertriangular relation on $\mathrm{X}^{2}$ to $\mathrm{U}$ for all $\mathrm{n} \in \mathbb{N}$.

Proof. If $n \in \mathbb{N}$, then by the corresponding definitions we have

$$
\begin{aligned}
(n F)(x, y)+(n F)(y, z) & =n F(x, y)+n F(y, z) \\
& =n(F(x, y)+F(y, z)) \subseteq n F(x, z)=(n F)(x, z)
\end{aligned}
$$

for all $x, y, z \in X$.

Remark 29 If $F$ is a supertriangular relation on $X^{2}$ to $\mathrm{U}$ and $\mathrm{U}$ has a zero element, then

$$
(\mathrm{OF})(x, y)=\emptyset \quad \text { if } \quad F(x, y)=\emptyset \quad \text { and } \quad(O F)(x, y)=\{0\} \quad \text { if } \quad F(x, y) \neq \emptyset .
$$

Therefore, $\mathrm{OF}$ is a supertriangular function on $\mathrm{X}^{2}$ to $\mathrm{U}$.

Now, analogously to Theorem 28, we can also prove the following

Theorem 29 If $\mathrm{F}$ is a supertriangular relation on $\mathrm{X}^{2}$ to $\mathrm{U}$ and $\mathrm{U}$ is a commutative group, then $\mathrm{kF}$ is also a supertriangular relation on $\mathrm{X}^{2}$ to $\mathrm{U}$ for all $k \in \mathbb{Z}$.

Moreover, in addition to Theorems 28, we can also easily prove the following

Theorem 30 If $\mathrm{F}$ and $\mathrm{G}$ are supertriangular relations on $\mathrm{X}^{2}$ to $\mathrm{U}$ and $\mathrm{U}$ is a commutative semigroup, then $\mathrm{F}+\mathrm{G}$ is also a supertriangular relation on $\mathrm{X}^{2}$ to $\mathrm{U}$.

Proof. By the corresponding definitions, it is clear that

$$
\begin{aligned}
& (F+G)(x, y)+(F+G)(y, z)=F(x, y)+G(x, y)+F(y, z)+G(y, z) \\
& =F(x, y)+F(y, z)+G(x, y)+G(y, z) \subseteq F(x, z)+G(x, z)=(F+G)(x, z)
\end{aligned}
$$

for all $x, y, z \in X$. 


\section{An application of the above results}

Now, by using Theorems 26, 27 and 30, we can also easily establish

Theorem 31 If $\xi$ a function of $\mathrm{X}$ to $\mathrm{U}, \mathrm{U}$ is a commutative group, $\mathrm{V}$ is a subgroupoid of $\mathrm{U}$ and

$$
F(x, y)=\xi(x)-\xi(y)+V
$$

for all $\mathrm{x}, \mathrm{y} \in \mathrm{X}$, then $\mathrm{F}$ is a supertriangular relation on $\mathrm{X}^{2}$ to $\mathrm{U}$ such that, under the notations of Definition 7 , for any $\mathrm{x}, \mathrm{y} \in \mathrm{X}$ we have:

(1) $\Phi(x)=V ; \quad(2) \quad S(x, y)=V+V$;

(3) $\Psi(x)=\emptyset$ if $X=\emptyset$ and $\Psi(x)=V+V$ if $X \neq \emptyset$.

Proof. From Theorems 26, 27 and 30, it is clear that $F$ is supertriangular. Moreover, by the corresponding definitions, it is clear that

$$
\begin{gathered}
\Phi(x)=F(x, x)=\xi(x)-\xi(x)+V=V, \\
S(x, y)=F(x, y)+F(y, x)=\xi(x)-\xi(y)+V+\xi(y)-\xi(x)+V=V+V
\end{gathered}
$$

and

$$
\Psi(x)=\bigcup_{y \in X} S(x, y)=\bigcup_{y \in X}(V+V)=\left\{\begin{array}{ccc}
\emptyset & \text { if } & X=\emptyset \\
V+V & \text { if } & X \neq \emptyset
\end{array}\right.
$$

Moreover, for an easy illustration of this theorem, we can also state

Example 4 If $r \geq 0$ and

$$
F(x, y)=[x-y+r,+\infty[
$$

for all $x, y \in \mathbb{R}$, then $\mathrm{F}$ is a supertriangular relation of $\mathbb{R}^{2}$ to $\mathbb{R}$ such that, for any $\mathrm{x}, \mathrm{y} \in \mathrm{X}$, we have:

(1) $\Phi(x)=[r,+\infty[$;

(2) $\Psi(x)=S(x, y)=[2 r,+\infty[$.

To check this, note that, by taking $\xi=\Delta_{\mathbb{R}}$ and $\mathrm{V}=[\mathrm{r},+\infty[$, we have

$$
F(x, y)=[x-y+r,+\infty[=x-y+[r,+\infty[=\xi(x)-\xi(y)+V
$$

for all $\mathrm{x}, \mathrm{y} \in \mathrm{X}$. Therefore, Theorem 31 can be applied.

For instance, by assertion (2) of Theorem 31, we have

$$
\mathrm{S}(x, y)=\mathrm{V}+\mathrm{V}=[\mathrm{r},+\infty[+[\mathrm{r},+\infty[=[2 r,+\infty[
$$

for all $\mathrm{x}, \mathrm{y} \in \mathrm{X}$. 
Remark 30 Note that in the present particular case, for any $x, y \in \mathbb{R}$, we have:

(1) $0 \in \Phi(x) \Longleftrightarrow r=0$;

(2) $\Phi(x)=\Psi(x) \Longleftrightarrow r=0$;

(3) $x-y \in F(x, y) \Longleftrightarrow r=0$;

(4) $0 \in \mathrm{F}(x, y) \Longleftrightarrow r \leq y-x$;

(5) $0 \in \mathrm{F}(x, y) \cap \mathrm{F}(\mathrm{y}, \mathrm{x}) \Longleftrightarrow \mathrm{r}=0, \quad x=y$.

To prove (5), note that by (4) we have

$0 \in F(x, y) \cap F(y, x) \Longleftrightarrow r \leq y-x, r \leq x-y \Longleftrightarrow r \leq \min \{x-y, y-x\}$.

Moreover, recall that $\min \{a, b\}=2^{-1}(a+b-|a-b|)$ for all $a, b \in \mathbb{R}$, and thus in particular $\min \{x-y, y-x\}=-|x-y|$. Therefore,

$$
r \leq \min \{x-y, y-x\} \Longleftrightarrow r \leq-|x-y| \Longleftrightarrow r=0, \quad x=y .
$$

\section{Acknowledgement}

The authors are indepted to the referee for suggesting grammatical corrections and stylistic changes.

\section{References}

[1] J. Aczél, Lectures on Functional Equations and Their Applications, Academic Press, New York, 1966.

[2] M. Alimohammady, S. Jafari, S. P. Moshokoa and M. K. Kalleji, A note on properties of hypermetric spaces, J. Hyperstructures, 3 (2014), 89-100.

[3] P. Augustová and L. Klapka, Atlas as solutions of Sincov's inequality, arXiv: 1612.00355v1 [math.DS] 1 Dec 2016, 7 pp.

[4] J. A. Baker, Solution of problem E 2607, Amer. Math. Monthly, 84 (1977), 824-825.

[5] G. Birkhoff, Lattice Theory, Amer. Math. Soc. Colloq. Publ., 25, Providence, Rhode Island, 1967.

[6] H. Brandt, Über eine Verallgemeinerung der Gruppenbregriffes, Math. Ann., 96 (1926), 360-366. 
[7] M. J. Campión, R. G. Catalán, E. Induráin and G. Ochoa, Reinterpreting a fuzzy subset by means of a Sincov's functional equation, J. Intelligent Fuzzy Systems, 27 (2014), 367-375.

[8] M. J. Campión, E. Induráin, G. Ochoa and O. Valero, Functional equations related to weightable quasi-metrics, Hacet. J. Math. Stat., 44 (2015), $775-787$.

[9] M. Cantor, Funktionalgleichungen mit drei von einander unabhängigen Veränderlichen, Zeitschrift Mat. Physik., 41 (1896), 161-163.

[10] E. Castillo-Ron and R. Ruiz-Cobo, Functional Equations in Science and Engineering, Marcel Decker, New York, 1992.

[11] R. Croisot, Une interprétation des relations d'équivalence dans un ensemble, C. R. Acad. Sci. Paris, 226 (1948), 616-617.

[12] B. A. Davey and H. A. Priestley, Introduction to Lattices and Order, Cambridge University Press, Cambridge, 2002.

[13] W. Fechner, Richard's inequality, Cauchy-Schwarz's inequality and approximate solutions of Sincov's equation, Proc. Amer. Math. Soc., 147 (2019), 3955-3960.

[14] W. Fechner, Sincov's inequalities on topological spaces, Publ. Math. Debrecen, 96 (2020), 63-76.

[15] G. Frege, Rechnungsmethoden, die sich auf eine Erweiterung des Grössenbegriffes gründen, Dissertation for the Venia docendi, Verlag Friedrich Frommann, Jena, 1874.

[16] G. Frege, Methods of calculation based on an extension of the concept of quantity, In: G. Frege, Collected Papers of on Mathematics, Logic, and Philosophy, Basil Blackwell, Oxford, 1984, 56-92.

[17] Z. Gajda, Invariant means and representations of semigroups in the theory of functional equations, Prace Naukowe Uniwersytetu Ślasskiego w Katowicach, 1273 (1992), 1-81.

[18] T. Glavosits, Generated preorders and equivalences, Acta Acad. Paed. Agriensis, Sect. Math., 29 (2002), 95-103. 
[19] T. Glavosits and Á. Száz, Constructions and extensions of free and controlled additive relations, In: Th.M. Rassias (Ed.), Handbook of Functional Equations: Functional Inequalities, Springer Optim. Appl. 95 (2014), 161-208.

[20] D. Gronau, Gottlob Frege, a pioneer in iteration theory, In: L. Reich, J. Smítal and Gy. Targonski (Eds.), Proceedings of the European Conference on Iteration Theory, ECIT94, Gracer Math. Ber. 334 (1997), 105-119.

[21] D. Gronau, Gottlob Fregees Beiträge zur Iteratiostheorie und zur Theorie der Functionalgleichungen, In: G. Gabriel (Ed.), Gottlob Frege - Werk und Wirkung, Mentis Verlag, Paderborn, 200, 151-169.

[22] D. Gronau, A remark on Sincov's functional equation, Notices South African Math. Soc. Esaim: Proceeding and Surveys, 31 (2000), 1-8.

[23] D. Gronau, Translation equation and Sincov's equation - A historical remark, Esaim: Proceeding and Surveys 46 (2014), 43-46.

[24] J. Mala and Á. Száz, Modifications of relators, Acta Math. Hungar., 77 (1997), 69-81.

[25] Z. Moszner, Solution générale de l'équation $F(x, y) F(y, z)=F(x, z)$ pour $\mathrm{x} \leq \mathrm{y} \leq z$, C. R. Acad. Sci. Paris, 261 (1965), 28.

[26] Z. Moszner, L'équation de translation et l1équation de Sincov généralisée, Rocznik Nauk.-Dydakt. Prace Mat., 16 (1999), 53-71.

[27] Z. Moszner, On the stability of functional equations, Aequationes Math., 77 (2009), 33-88.

[28] K. Nikodem, Additive selections of additive set-valued functions, Zb. Rad. Prirod.-Mat. Fak., 18 (1988), 143-148.

[29] K. Nikodem and D. Popa, On single-valuedness of set-valued maps satisfying linear inclusions, Banach J. Math. Anal., 3 (2009), 44-51.

[30] G. Pataki and Á. Száz, A unified treatment of well-chainedness and connectedness properties, Acta Math. Acad. Paedagog. Nyházi. (N.S.), 19 (2003), 101-165.

[31] J. Pepis, Sur une famille d'ensembles plans et les solutions de l'équation fonctionnelle $\mathrm{F}(x, z)=\mathrm{F}(x, y) \cdot \mathrm{F}(\mathrm{y}, z)$ pour $0 \leq x \leq y \leq z$. Application á la théorie générale des intéréts, Ann. Soc. Polon. Math., 17 (1937), 113. 
[32] W. J. Pervin, Quasi-uniformization of topological spaces, Math. Ann., 147 (1962), 316-317.

[33] B. Piatek, On the Sincov functional equation, Demonstratio Math., 38 (2005), 875-881.

[34] P. K. Sahoo, Stability of a Sincov type functional equation, J. Inf. Math. Sci., 1 (2009), 81-90.

[35] P. K. Sahoo, On a Sincov type functional equation, In: Th.M. Rassias and J. Brzdek (Eds.), Functional Equations in Mathematical Analysis, Springer Optimizations and Its Applications, 52, Chapter 43, Springer, New York, 2012, 697-7008.

[36] D. M. Sincov, Über eine Funktionalgleichung, Arch. Math. Phys., 6 (1904), 216-217.

[37] A. Smajdor, Iterations of multi-valued functions, Prace Naukowe Universytetu Ślaskiego w Katowicach 759, Universitet Ślaski, Katowice 1985.

[38] W. Smajdor, Set-valued version of Sincov's functional equation, Demonstration Math., 39 (2006), 101-105.

[39] Á. Száz, Galois type connections and closure operations on preordered sets, Acta Math. Univ.Comen., 78 (2009), 1-21.

[40] Á. Száz, Corelations are more powerful tools than relations, In: Th. M. Rassias (Ed.), Applications of Nonlinear Analysis, Springer Optimization and Its Applications, 134 (2018), 711-779.

[41] A. Weil, Sur les espaces á structure uniforme at sur la topologie générale, Actual. Sci. Ind., 551 Herman and Cie, Paris, 1937.

Received: January 17, 2019 